Sonderdruck aus

\title{
ABHANDLUNGEN
}

AUS DEM

\section{MATHEMATISCHEN SEMINAR} DER

\section{UNIVERSITÄT HAMBURG}

\section{Herausgegeben von}

H. Bauer · L. Collatz · H. Hasse · E. Kähler

E. Sperner - E. Witt

Band 28, Heft 3/4

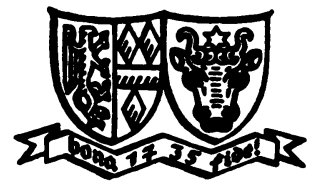

Oktober 1965

VANDENHOEGK \& RUPREGHT IN GOTTINGEN 


\section{BAND 28, HEFT $3 / 4$}

\section{Inhalt}

A. Dress, Eine Bemerkung über Teilringe globaler Körper .......... 134

G. Ringel, Das Geschlecht der vollstëndigen paren Graphen ......... 139

K. Hoechsmann, Uber einen Satz von Teichmüller ............ 151

P. X. Gallagher, Determinants of Representations of Finite Groups . . . . 162

M. Knebusch, Der Begriff der Ordnung in einer Jordanalgebra . . . . . . . 168

M. Knebusch, Eine Klasse von Ordnungen in Jordanalgebren vom Grade 3185

G. Harder, Uber einen Satz von E. Cartan ................. 208

H. Leptin, Ideale endlicher Codimension in $\mathrm{L}^{1}$-Algebren........... 215

H. Zieschang, Alternierende Produkte in freien Gruppen, II.......... 219

K. Soltsien, Bestimmung von Schlingknoten ................. 232

H. Salzmann, Zur Klassifikation topologischer Ebenen ............. 250 



\title{
Der Begriff der Ordnung einer Jordanalgebra
}

\author{
Von MANFRed Knebusch in Hamburg
}

\section{Einleitung}

Weil die Jordanalgebren potenzassoziativ sind, lassen sich in ihnen ebenso wie in assoziativen Algebren ganze Elemente definieren, sofern im Grundkörper schon ganze Elemente erklärt sind. Damit stoßen wir auf die Frage nach einer Zahlentheorie in Jordanalgebren.

Bei der Entwicklung einer Arithmetik für nicht kommutative, assoziative Algebren stellte sich als Schwierigkeit heraus, daß Summe und Produkt zweier ganzer Elemente nicht mehr ganz zu sein brauchen. Man muß daher zuerst, wie schon Dickson erkannte, maximale Ordnungen, d.h. möglichst große gegen Addition und Multiplikation abgeschlossene Bereiche ganzer Elemente aufsuchen (s. [4] u.d. angeg. Literatur). Dieses Vorgehen erwies sich als zweckmäßig nicht nur bei den assoziativen, sondern z.B. auch bei den alternativen einfachen Algebren (s. [13] u. d. angeg. Literatur).

Für eine einfache Jordanalgebra $\mathfrak{A}$ über dem Quotientenkörper eines Dedekindringes kommt zu dem Umstand, daß Summe und Produkt ganzer Elemente im allgemeinen nicht ganz sind, eine weitere Schwierigkeit hinzu: Ist $x$ ganzes Element der Algebra, d.h. besitzt $x$ nur ganze Eigenwerte, so kann doch bei den Eigenwerten der linearen Transformation $z \rightarrow x z$ von $\mathfrak{A}$ in sich die Zahl 2 als Nenner auftreten. Dieser Umstand veranlaßte den Verfasser, für eine Ordnung $M$ an Stelle der Abgeschlossenheit gegen Multiplikation die etwas allgemeinere Bedingung

$$
x \in M, y \in M \Rightarrow P(x) y:=2 x(x y)-x^{2} y \in M
$$

zu fordern. Der von N. Jacobson ([6]) und M. Komcher ([12]) eingeführte Operator $P(x)$ besitzt grundlegende Bedeutung für die algebraische Theorie der Jordanalgebren (s. [10], [8], [3]). Ist $x$ ganz, so sind auch alle Eigenwerte von $P(x)$ ganz (s. [11], S. 92). Ist die Zahl 2 Einheit, so ist (*) mit der Abgeschlossenheit von $M$ gegen Multiplikation gleichwertig, sofern man für $M$ die Abgeschlossenheit gegen Addition schon voraussetzt (s. § 2).

In § 2 dieser Arbeit wird der so entstehende Begriff „Ordnung“ an Hand einfacher Konsequenzen motiviert. In § 3 zeigen wir, daß alle 
ganzen Elemente eines lokalen Jordankörpers eine Ordnung in dem hier definierten Sinne bilden, in Parallele zu einem bekannten Satz der assoziativen Theorie. In $\S 4$ gehen wir kurz auf den Zusammenhang unseres Begriffes „Ordnung" für Jordanalgebren vom Grade 2 mit der Arithmetik der quadratischen Formen ein. Die zum Verständnis der Arbeit erforderlichen Resultate aus der algebraischen Theorie der Jordanalgebren - entwickelt vor allem von A. A. ALBERT und N. JACOBson werden in $\S 1$ formuliert.

Das sich jetzt stellende Problem, eine Utbersicht über die Klassen isomorpher maximaler Ordnungen einer einfachen Jordanalgebra zunächst mit lokalem Grundkörper - zu gewinnen, läßt sich für den Grad 2 ziemlich leicht lösen (s. [14]). In seiner Dissertation hat der Verfasser für lokale Algebren vom Grad 3 die ausgezeichneten Ordnungen klassifiziert, d.h. diejenigen Ordnungen, die von keinem Gitter ganzer Elemente echt umfaßt werden. Bei gewissen Algebrentypen mußte er allerdings voraussetzen, daß der Grundkörper nicht verzweigt dyadisch ist. Die Veröffentlichung dieser Resultate ist geplant. In Fortsetzung der vorliegenden Arbeit werden in [9] Sätze bewiesen, die sie vorbereiten.

Ich gedenke dankbar meines verehrten Lehrers EMrL ARTrN. Er hat mich zu der Beschäftigung mit zahlentheoretischen Problemen in Jordanalgebren angeregt. Weiter gehört mein Dank Frau HrL. BraUn, die mich, besonders nach dem Tode ARTINs, mit manchem Ratschlag unterstützt hat.

\section{\$1. Benutzte algebraische Begriffe und Resultate}

Alle hier mitgeteilten Sachverhalte findet man in [1], [3], [8]. Eine Jordanalgebra $\mathfrak{A}$ ist eine kommutative Algebra mit

$$
x^{2}(x z)=x\left(x^{2} z\right)
$$

für beliebige $x, z \in \mathfrak{A}$. Der Grundkörper $k$ habe grundsätzlich eine Charakteristik $\neq 2$. $\mathfrak{A}$ soll in dieser Arbeit stets ein Einselement $e$ besitzen.

Wir definieren zu einem $x \in \mathfrak{A}$ sukzessive die Potenzen

Es gilt dann

$$
x^{0}=e, \quad x^{1}=x, \quad x^{n}=x\left(x^{n-1}\right) .
$$

$$
\begin{aligned}
& x^{n} x^{m}=x^{n+m} \quad \text { (s. AlBERT [1]). } \\
& k[x]:=\sum_{u \geq 0} k x^{n}
\end{aligned}
$$

ist also eine assoziative und kommutative Algebra. Deshalb hat es Sinn, ein Element $z$ von $\mathfrak{A}$ in ein Polynom $g(\tau) \in k[\tau]$ einzusetzen und von $\operatorname{dem}$ Minimalpolynom $f(\tau ; z)$ von $z$ zu reden. Der Grad von $f(\tau ; z)$ ist 
die Dimension von $k[x]$ über $k$. Somit ändert sich dieser Grad, also auch $f(\tau ; z)$ selbst, nicht bei Grundkörpererweiterung.

$\mathbb{Z u}$ einem $x \in \mathfrak{A}$ definieren wir auf $\mathfrak{A}$ den Operator $L(x): x \rightarrow x z$. Zwei Elemente $x, y$ aus $\mathfrak{A}$ heißen vertauschbar, wenn $L(x) L(y)$ $=L(y) L(x)$ ist. Nach (1.1) ist $x$ mit $x^{2}$ vertauschbar. Es sind aber sogar beliebige Elemente von $k[x]$ miteinander vertauschbar (8. ALBERT [1]).

Als Zentrum unserer Algebra bezeichnet man die Menge der $z \in \mathfrak{A}$, die mit allen Elementen von $\mathfrak{A}$ vertauschbar sind. Es ist eine assoziative und kommutative Algebra, die das Einselement enthält.

In $\mathfrak{A}$ definieren wir neben $L(x)$ den Operator

$$
P(x):=2 L^{2}(x)-L\left(x^{2}\right) .
$$

Es gibt gewichtige Gründe, nicht $L(x)$, sondern $P(x)$ als Gegenstück zu den regulären Darstellungen bei assoziativen Algebren anzusehen.

Für $P(x)$ gilt die „Fundamentalformel“

$$
P(P(x) y)=P(x) P(y) P(x) .
$$

Ferner ist, falls $x, y, x y$ paarweise vertauschbar sind.

$$
P(x y)=P(x) P(y) .
$$

Zur Theorie des von N. JACoBson [6] eingeführten Operators $P(x)$ s. KOFCHFr [10] und die dort angegebene Literatur.

Sei E eine assoziative Algebra, deren Multiplikation wir mit $a \cdot b$ bezeichnen. Führt man auf \& die neue Verknüpfung

$$
a b=\frac{1}{2}(a \cdot b+b \cdot a)
$$

ein, so erhält man, wie leicht nachgerechnet werden kann, eine Jordanalgebra, die wir mit $F^{+}$bezeichnen. Solche Algebren (E+ und ihre JordanTeilalgebren heißen spezielle Jordanalgebren. Für den Operator (1.3) erhält man in E+ ohne Mühe:

$$
P(x) y=x \cdot y \cdot x .
$$

(1.7) macht ein wenig plausibel, warum der Operator $P(x)$ in der Theorie der Jordanalgebren eine dominierende Rolle spielt.

Ein Element $x \in \mathfrak{A}$ heißt invertierbar, wenn ein mit $x$ vertauschbares $y$ existiert mit $x y=e$. Dieses Element $y$ ist dann eindeutig bestimmt und liegt in $k[x]$. Wir schreiben $y=x^{-1} . x \in \mathfrak{A}$ ist also genau dann invertierbar, wenn $x$ nicht Nullteiler in $k[x]$ ist. Aus (1.5) liest man weiter ab, daß $x$ genau dann invertierbar ist, wenn $P(x)$ invertierbar ist, und daß dann

$$
P\left(x^{-1}\right)=P(x)^{-1}
$$

gilt. 
Sei $x=\Sigma \varrho_{i} a_{i}\left(a_{1}, \ldots, a_{n}\right.$ Basis von $\mathfrak{A}, \varrho_{1}, \ldots, \varrho_{n}$ Unbestimmte) generisches Element von $\mathfrak{A}$. Das Minimalpolynom von $x$ in der Grundkörpererweiterung $\mathfrak{A}_{\tilde{k}}$ mit $\tilde{k}=k\left(\varrho_{1}, \ldots, \varrho_{n}\right)$ schreiben wir in der Form

$$
f(\tau ; x)=\tau^{n}-\sigma_{1}(x) \tau^{n-1}+\cdots+(-1)^{n} \sigma_{n}(x) .
$$

Die Gleichung $x^{n}-\sigma_{1}(x) x^{n-1}+\cdots+(-1)^{n} \sigma_{n}(x)=0$ dazu heißt die Minimalgleichung von $\mathfrak{A}, n$ heißt der $\operatorname{Grad}$ von $\mathfrak{A} . \sigma_{i}(x)$ ist homogenes Polynom vom Grade $i$ auf dem Vektorraum $\mathfrak{A}$ (s. [7], [8], [3]).

Besonders wichtig unter den $\sigma_{i}(x)$ sind

$$
\begin{array}{ll}
M S(x):=\sigma_{1}(x) & \text {,Minimalspur", } \\
M N(x):=\sigma_{n}(x) & \text {,Minimalnorm“. }
\end{array}
$$

Mit Hilfe von $M N$ läßt sich das Polynom (1.8) in der Form $M N(\tau e-x)$ schreiben. Wir notieren:

(1.9) $M N(\tau e-x)=\tau^{n}-M S(x) \tau^{n-1}+\sigma_{2}(x) \tau^{n-2}+\cdots+(-1)^{n} M N(x)$.

Eigenschaften von $M N$ und $M S$ (s. [2], [3], [8]):

$$
\begin{aligned}
M N(P(x) y) & =M N^{2}(x) M N(y) \quad(x, y \text { beliebig in } \mathfrak{A}) . \\
M N(x y) & =M N(x) M N(y),
\end{aligned}
$$

sofern $x, y$ vertauschbar sind oder in einem assoziativen Teilring von $\mathfrak{A}$ liegen.

$$
M N(x+y)=M N(x) M N(y) M N\left(x^{-1}+y^{-1}\right)
$$

für invertierbare $x, y \in \mathfrak{A}$.

$$
M S((x y) z)=M S(x(y z)) \text { für beliebige } x, y, z \in \mathfrak{A} \text {. }
$$

Sei $z$ ein Element von $\mathfrak{A}$. Ein Element $\zeta$ aus dem algebraischen Abschluß $\bar{k}$ von $k$ heißt Eigenwert von $z$, wenn in $\mathfrak{A}_{\bar{k}}$ ein mit $z$ vertauschbarer Vektor $v \neq 0$ existiert, für den

$$
z \boldsymbol{v}=\zeta v
$$

ist. $v$ heißt ein zu $\zeta$ gehöriger Eigenveltor von $z$.

Wir nennen einige wichtige Tatsachen über Eigenwerte:

(1.15) 1. $\mathrm{Zu}$ jedem Eigenwert $\zeta \in \bar{k}$ von $z$ gibt es sogar in $\bar{k}[z]$ einen Eigenvektor.

(1.16) 2. Die Nullstellen des Minimalpolynoms $f(\tau ; z)$ wie des dadurch teilbaren Polynoms $M N(\tau e-z)$ sind sämtliche Eigenwerte von $z$ in gewissen (positiven) Vielfachheiten. Wir bezeichnen als Vielfachheit eines Eigenwertes $\zeta$ von $z$ - nicht ganz konform mit [3] - die Vielfachheit der Nullstelle $\zeta$ von $M N(\tau e-z)$. 
(1.17) 3. $\zeta_{1}, \ldots, \zeta_{n}$ seien die Eigenwerte von $z$ unter Berücksichtigung ihrer Vielfachheit. $g(\tau)$ sei beliebiges Polynom aus $k[\tau]$. Dann sind die Eigenwerte von $g(z)$ unter Berücksichtigung ihrer Vielfachheit $g\left(\zeta_{1}\right), \ldots$, $g\left(\zeta_{n}\right)$.

Aus (1.16) ergibt sich

$$
M N(\tau e-z)=\prod_{i=1}^{n}\left(\tau-\zeta_{i}\right)
$$

(1.18) zeigt, daß die $\sigma_{i}(z)$ die elementarsymmetrischen Funktionen der Eigenwerte $\zeta_{1}, \ldots, \zeta_{n}$ von $z$ sind. Insbesondere ist

$$
\begin{aligned}
& M S(z)=\sum_{i=1}^{n} \zeta_{i}, \\
& M N(z)=\prod_{i=1}^{n} \zeta_{i} .
\end{aligned}
$$

Weil $z^{k}$ die Eigenwerte $\zeta_{1}^{k}, \ldots, \zeta_{n}^{k}$ besitzt, ist nach (1.19)

$$
M S\left(z^{k}\right)=\sum_{i=1}^{n} \zeta_{i}^{k}
$$

Aus der elementaren Algebra ist bekannt, daß sich die $\sigma_{i}(z)$ durch die $M S\left(z^{k}\right)$ ausdrücken lassen (und umgekehrt). Insbesondere ist

$$
\sigma_{2}(z)=1 / 2\left(M S^{2}(z)-M S\left(z^{2}\right)\right)
$$

und falls die Charakteristik von $k$ auch $\neq 3$ ist:

$$
\sigma_{3}(z)=1 / 6\left\{M S^{3}(z)-3 M S(z) M S\left(z^{2}\right)+2 M S\left(z^{3}\right)\right\}
$$

(Ist $n<3$, so wird $\sigma_{3}(z)$ zu Null festgesetzt.)

Im Anschluß an (1.20) geben wir noch ein Kriterium für die Invertierbarkeit eines Elementes $z$ von $\mathfrak{A}$ an. $z$ ist genau dann invertierbar, wenn $z$ nicht den Eigenwert Null besitzt, wie man mit (1.15) sofort sieht. Also gilt:

$$
z \text { invertierbar } \leftrightarrow M N(z) \neq 0 .
$$

Eine Jordanalgebra $\mathfrak{A}$ heißt einfach, wenn sie außer 0 und $\mathfrak{A}$ keine Ideale besitzt und $\mathfrak{A} \mathfrak{A} \neq 0$ ist. (Ideal $\mathfrak{B}$ von $\mathfrak{A}: \mathfrak{B}+\mathfrak{B} \subset \mathfrak{B}, \mathfrak{A} \mathfrak{B} \subset \mathfrak{B}$.) Eine einfache Jordanalgebra besitzt stets ein Einselement. Ihr Zentrum ist ein Körper. Sie heißt zentral einfach, wenn dieser Körper $k e$ ist.

Eine Jordanalgebra mit Einselement heißt nicht ausgeartet, wenn die Bilinearform $M S(x y)$ nicht ausgeartet ist. Es läßt sich zeigen, daß die nicht ausgeanteten Algebren genau die direkten Summen von einfachen Algebren sind, deren Zentren über $k$ separable Körper sind. 


\section{§ 2. Ganze Elemente, Ordnungen}

$\mathfrak{A}$ sei von jetzt an stets eine nichtausgeartete Jordanalgebra uber dem Quotientenkörper $k$ eines Dedekindringes $A$. Die Elemente von $A$ bezeichnen wir als ,ganze Elemente" von $k$.

Ein Element $z$ von $\mathfrak{A}$ heiße ganz, wenn es einer Gleichung

$$
z^{r}+\alpha_{1} z^{r-1}+\cdots+\alpha_{r} e=0
$$

mit $\alpha_{1}, \ldots, \alpha_{r} \in A$ genügt. Dies ist offenbar gleichwertig mit der Aussage, daß $A[z]:=\sum_{\nu \geq 0} A z^{\nu}$ ein endlich erzeugter $A$-Modul ist.

Aus (2.1) folgt nach (1.17) für einen Eigenwert $\zeta \in \bar{k}$ von $z$ :

$$
\zeta^{r}+\alpha_{1} \zeta^{r-1}+\cdots+\alpha_{r}=0 .
$$

Die Eigenwerte von $z$ sind also ganze algebraische Elemente über $k$. Umgekehrt folgt aus dieser Eigenschaft, da $B$ alle Koeffizienten des Minimalpolynoms $f(\tau ; z)$ von $z$ wie des Polynoms $M N(\tau e-z)$ in $A$ liegen.

Wir fassen zusammen:

Satz 2.1. Ein Element $z$ von $\mathfrak{A}$ ist genau dann ganz, wenn eine der folgenden Bedingungen gilt:

(1) $A[z]$ ist endlich erzeugter $A$-Modul.

(2) Alle Eigenwerte von $z$ sind ganze algebraische Elemente uber $k$.

(3) Das Minimalpolynom von $z$ hat ganze Koeffizienten.

(4) $\sigma_{i}(z) \in A$ fur $i=1, \ldots, n$.

Unsere Definition von ganzen Elementen in $\mathfrak{A}$ ist sehr naheliegend: Ein Element $z$ aus $\mathfrak{A}$ liegt in der assoziativen und kommutativen Algebra $k[z]$. Der für solche Algebren geläufige Ganzheitsbegriff wird übertragen. Bevor wir den Begriff der „Ordnung" einführen, beweisen wir noch

Satz 2.2. $M$ sei ein $A$-Modul in $\mathfrak{A}$, der nur ganze Elemente enthält.

Behauptung. Far zwei beliebige $x, y$ von $M$ gilt

$$
M S(x y) \in A
$$

und, falls die Charakteristik von $k$ auch $\neq 3$ ist,

$$
\left.M S\left(x^{2} y\right)+M S\left(x y^{2}\right) \in A^{1}\right) .
$$

Beweis. Für jedes $x \in M$ liegt

$$
\sigma_{2}(x)=1 / 2\left(M S^{2}(x)-M S\left(x^{2}\right)\right)
$$

1) (2.3) wird erst in [9] gebraucht. 
in $A$. Polarisation liefert:

$$
M S(x) M S(y)-M S(x y) \in A \quad \text { für } x, y \in M .
$$

Daraus folgt wegen $M S(x), M S(y) \in A$ die Behauptung (2.2). Zum Beweis von (2.3) betrachten wir (s. (1.23))

$$
\sigma_{3}(x)=1 / 6\left\{M S^{3}(x)-3 M S(x) M S\left(x^{2}\right)+2 M S\left(x^{3}\right)\right\},
$$

das für jedes $x \in M$ in $A$ liegt. Setzt man für $x$ das Element $x+y$ ein $(x \in M, y \in M)$ und sammelt die ,gemischten Glieder", so erhält man mit (1.13):

$$
\begin{gathered}
1 / 6\left\{3 M S^{2}(x) M S(y)+3 M S(x) M S^{2}(y)-3 M S(x) M S\left(y^{2}\right)\right. \\
-3 M S(y) M S\left(x^{2}\right)-6 M S(x) M S(x y)-6 M S(y) M S(x y) \\
\left.+6 M S\left(x^{2} y\right)+6 M S\left(x y^{2}\right)\right\} \in A,
\end{gathered}
$$

also weiter, unter Beachtung von (2.2):

$$
\begin{aligned}
1 / 2\left(M S^{2}(x)-M S\left(x^{2}\right)\right) M S(y) & +1 / 2\left(M S^{2}(y)-M S\left(y^{2}\right)\right) M S(x) \\
& +M S\left(x^{2} y\right)+M S\left(x y^{2}\right) \in A .
\end{aligned}
$$

Daraus folgt (2.3).

q. e. d.

Bemerkung. Behandelt man in entsprechender Weise die Aussagen $\sigma_{i}(x) \in A$ für $i \geqq 4$, so erhält man erheblich kompliziertere Beziehungen, weil dann (1.13) kaum ausgenutzt werden kann.

Aus (2.2) ergibt sich unmittelbar

Lemma 2.1. $M$ sei ein $A$-Modul von ganzen Elementen, der $\mathfrak{A}$ aufspannt $(k M=\mathfrak{A})$.

Behauptung. $M$ ist endlich erzeugter $A$-Modul. (Ein endlich orzeugter $\boldsymbol{A}$-Teilmodul von $\mathfrak{A}$, der $\mathfrak{A}$ aufspannt, heißt Gitter von $\mathfrak{A}$.)

Beweis. $M$ enthält eine Basis $x_{1}, \ldots, x_{\star}$ des $k$-Vektorraumes $\mathfrak{A}$, also das Gitter

$$
N=\sum_{i=1}^{\infty} A x_{i}
$$

Nach (2.2) ist $M$ in

(2.4) $\tilde{N}:=\{x \in \mathfrak{A} \mid M S(x y) \in A$ für alle $y \in N\}$ enthalten.

Es ist $\tilde{N}=\sum A y_{i}$, wobei $\left\{y_{i}\right\}$ die zu $\left\{x_{j}\right\}$ duale Basis bezeichne, definiert durch

$$
M S\left(x_{i} y_{j}\right)=\delta_{i j} .
$$

$N$ ist also endlich erzeugt. Weil $A$ noethersch ist, ist der Teilmodul $M$ von $\tilde{N}$ ebenfalls endlich erzeugt. q. e. d. 
Bemerkung. Der zu einem Gitter $N$ vermöge (2.4) definierte Modul $\tilde{N}$ ist bekanntlich auch, falls $N$ über $A$ keine Basis besitzt, ein Gitter und heißt das $z u N$ duale Gitter. In der Tat: $N$ enthält ein Gitter $S$ mit Basis. Es ist $\tilde{N} \subset \tilde{S}$. Daher ist mit $\tilde{S}$ auch $\tilde{N}$ endlich erzeugt.

Als Ordnung von $\mathfrak{A}$ definieren wir ein Gitter $M$ von $\mathfrak{A}$ mit

$$
\begin{gathered}
e \in M, \\
x, y \in M \Rightarrow P(x) y \in M .
\end{gathered}
$$

Zur Motivation von (2.6) bemerken wir zunz̈ohst folgendes:

Ist (E) assoziative Algebra, $M$ eine assoziative Ordnung von E $(x, y \in M \Rightarrow x \cdot y \in M)$, so ist $M$ nach (1.7) auch Ordnung von $E^{+}$. Hingegen gilt in $M$, wenn 2 nicht Einheit ist, im allgemeinen nicht

$$
x, y \in M \Rightarrow x y \in M \text {. }
$$

Beispiel. $A$ sei der Ring $Z$ der ganzen Zahlen, $k$ somit der Körper $Q$ der rationalen Zahlen. $\mathfrak{A}$ sei die Hamiltonsche Quaternionenalgebra $Q+Q i+Q j+Q k$ über $Q, \quad\left(i^{2}=j^{2}=-1, \quad i j=-j i=k\right), \quad M$ die Ordnung $Z i+Z j+Z k+Z g$ mit $g=1 / 2(1+i+j+k)$.

Es ist $1 / 2(i \cdot g+g \cdot i)=1 / 2(i-1) \notin M$.

(2.7) ist eine stärkere Bedingung als (2.6).

Lemma 2.2. Eine Ordnung $M$ von $\mathfrak{A}$ enthält mit einem Element $x$ auch alle Potenzen $x^{n}(n \geqq 0)$. M enthäl nur ganze Elemente.

Beweis. Es ist $x^{0}=e \in M, x^{1}=x \in M$. Sei $x^{n-1} \in M, x^{n} \in M$ schon bewiesen. Dann liegen auch

$$
x^{n+1}=P(x) x^{n-1}, \quad x^{n+2}=P(x) x^{n} \text { in } M .
$$

Es ist also $A[x] \subset M$. Weil $M$ endlich erzeugter $A$-Modul ist, gilt gleiches für $A[x] . x$ ist ganz.

q. e.d.

Wir werden in dieser und einer weiteren Arbeit [9] zeigen, daß für einfache Jordanalgebren vom Grade 2 und 3 bei unserer Definition von „Ordnung" statt (2.6) die Bedingung

$$
x \in M \Rightarrow x^{2} \in M
$$

herangezogen werden kann. Auf Grund von Lemma 2.2 erscheint (2.8) als Abschwächung von (2.6).

Ist 2 Einheit von $A$ so sind alle drei Bedingungen (2.8), (2.6), (2.7) gleichwertig. Aus (2.8) folgt ja

$$
x, y \in M \Rightarrow 2 x y \in M .
$$


Wir nennen eine Ordnung maximal, wenn sie von keiner Ordnung echt umfaßt wird. Wie in nichtausgearteten assoziativen Algebren gilt auch hier

Satz 2.3. Über jeder Ordnung gibt es eine maximale Ordnung.

Beweis. $M$ sei vorgegebene Ordnung von $\mathfrak{A}$. Gäbe es keine $M$ umfassende maximale Ordnung, so gäbe es eine nicht abbrechende Kette von Ordnungen $M_{i}$ über $M$ :

$$
M \underset{\ddagger}{\subset} M_{1} \underset{\ddagger}{\subset} M_{2} \subsetneq \ldots
$$

Alle diese $M_{i}$ liegen nach (2.2) in dem zu $M$ dualen Gitter $\mathscr{I}$. (2.9) steht im Widerspruch dazu, daß $\mathscr{I}$ noetherscher $A$-Modul ist.

$$
\text { q.e.d. }
$$

Satz 2.4. Jedes ganze Element von $\mathfrak{A}$ liegt in einer Ordnung von $\mathfrak{A}$.

Bemerkung. Hätten wir für den Begriff „Ordnung“" statt (2.6) die Bedingung (2.7) zugrunde gelegt, so wäre Satz $2.4 \mathrm{im}$ allgemeinen falsch, wie folgendes Beispiel zeigt: 2 sei nicht Einheit von $A, \mathfrak{A}$ sei eine einfache Jordanalgebra über $k$, die ein Idempotent $u \neq e$ besitzt $\left(u^{2}=u \neq 0\right)$, $M$ eine Ordnung von $\mathfrak{A}$, die sogar (2.7) genügt. Dann liegt das ganze Element $u$ nicht in $M$. $\mathfrak{A}$ enthält nämlich ein $x \neq 0$ mit $u x=1 / 2 x$ (s. AlbeRT [1]). Wir können $x$ als primitiven Vektor aus $M$ wählen, d.h. als Element von $M$ mit der Eigenschaft $\lambda \in k, x \in M \Rightarrow \lambda \in A$. Es ist $u x \notin M$, also wegen (2.7) auch $u \notin M$.

Beweis von Satz 2.4:

a) Zunächst merken wir an: Für einen endlich erzeugten $A$-Modul $N=\sum_{i=1}^{b} A x_{i} \subset \mathfrak{A}$ ist der $A$-Modul $P(N)$, den die Operatoren $P(n), n \in N$ in der Algebra der linearen Transformationen von $\mathfrak{A}$ erzeugen, wieder endlich erzeugt, z.B. von den Operatoren $P\left(x_{i}\right), P\left(x_{i}+x_{j}\right)-P\left(x_{i}\right)-P\left(x_{j}\right)$ $(i, j=1, \ldots, r, i \neq j)$.

b) Sei $x \in \mathfrak{A}$ ganz. $R:=P(A[x])$ ist nach a) endlich erzeugter $A$ Modul und auf Grund von (2.6) (kommutativer) Ring. Es gibt ein unter $R$ stabiles Gitter $M^{\prime}$ von $\mathfrak{A}$, z.B. $M^{\prime}=\sum R z_{i}$ mit irgendeiner Basis $z_{1}, \ldots, z_{\text {s }}$ von $\mathfrak{A}$ über $k$.

c) $M^{\prime} M^{\prime}$ und $P\left(M^{\prime}\right) A[x]$ sind endlich erzeugte $A$-Moduln. Daher existiert ein $\lambda \in k^{*}$ mit $M^{\prime} M^{\prime} \subset \lambda M^{\prime}, P\left(M^{\prime}\right) A[x] \subset \lambda M^{\prime}$. Für $M:=\lambda^{-1} M^{\prime}$ gilt neben $P(A[x]) M \subset M$ auch $M M \subset M, P(M) A[x] \subset M$. 
d) Wir zeigen jetzt: $B:=A[x]+M$ ist eine Ordnung. Sei $z \in A[x]$, $m \in M$. Es ist

$$
P(z+m)=P(z)+P(m)+2[L(z) L(m)+L(m) L(z)-L(z m)] .
$$

Aus c) ergibt sich sofort: $P(z) B C B, P(m) B C B$.

Weiter ist

$$
2 L(z)=P(e+z)-P(e)-P(z) \in P(A[x])
$$

und somit $2 z M \subset M$. Es folgt nun

$$
2[L(z) L(m)+L(m) L(z)-L(z m)] M \subset M .
$$

Für $y \in A[x]$ ist schließlich

$$
\begin{aligned}
& 2[L(z) L(m)+L(m) L(z)-L(z m)] y \\
& \quad=2 z(y m)+2 y(z m)-2(y z) m+4(y z) m-4 y(z m) \\
& \quad \equiv 2 z(y m)+2 y(z m)-2(y z) m \\
& \quad=[P(y+z)-P(y)-P(z)] m \equiv 0 \bmod M .
\end{aligned}
$$

Für $a, b \in B$ gilt also in der Tat $P(a) b \in B$,

q. e.d.

\section{§ 3. Jordankörper mit komplettem diskret bewertetem Grundkörper}

$k$ sei jetzt kompletter diskret bewerteter Körper. Wir können die Bewertung || von $k$ in genau einer Weise auf den algebraischen Abschluß $\bar{k}$ von $k$ fortsetzen. Diese Fortsetzung bezeichnen wir wieder mit || . Utber $k$ konjugierte Elemente von $\bar{k}$ haben gleichen Betrag.

$\mathfrak{A}$ sei Jordankörper über $k$, d.h. eine Jordanalgebra mit Einselement, in der jedes von Null verschiedene Element invertierbar ist. Für beliebiges $z$ aus $\mathfrak{A}$ ist $k[z]$ nullteilerfrei. Daher ist das Minimalpolynom $f(\tau ; z)$ von $z$ irreduzibel. Seine Nullstellen, die Eigenwerte von $z$, haben also gleichen Betrag.

Lemma 3.1. $k$ sei diskret und komplett bewertet. $\mathfrak{A}$ sei Jordankörper über k. Dann gilt für ein Element $z$ von $\mathfrak{A}$ :

$$
z \operatorname{ganz} \Leftrightarrow M N(z) \in A .
$$

Beweis. $M N(z)$ ist das Produkt der Eigenwerte $\zeta_{1}, \ldots, \zeta_{n}$ von $z$ unter Berücksichtigung ihrer Vielfachheit (s. (1.20)). Daher ist

Man sieht:

$$
|M N(z)|=\left|\prod_{i=1}^{n} \zeta_{i}\right|=\left|\zeta_{1}\right|^{n}
$$

$$
M N(z) \in A \Leftrightarrow\left|\zeta_{1}\right|=\cdots=\left|\zeta_{n}\right| \leqq 1 \Leftrightarrow z \operatorname{ganz}(\text { nach Satz 2.1). }
$$


Satz 3.1. $k$ sei diskret und komplett bewertet. $\mathfrak{A}$ sei Jordankörper aber $k$.

Behauptung. Die Menge der ganzen Elemente von $A$ ist eine Ordnung, somit die einzige maximale Ordnung von $A$.

Beweis. $x, y$ seien beliebige Elemente von $\mathfrak{A}$. Nach (1.10) ist

$$
M N(P(x) y)=M N(x)^{2} M N(y) .
$$

Man liest mit (3.1) ab:

$$
x, y \text { ganz } \Rightarrow P(x) y \text { ganz. }
$$

Etwas schwieriger ist es, zu zeigen, daß auch $x+y$ ganz ist. Es sei ohne Einschränkung der Allgemeinheit $x \neq 0, y \neq 0$ und $|M N(y)| \leqq|M N(x)|$.

Man erweitere $\mathfrak{A}$ durch Grundkörpererweiterung zu $\mathfrak{A}:=\mathfrak{A}_{\overline{\mathfrak{k}}} \cdot \mathfrak{A}$ ist gemeinhin kein Jordankörper mehr. Die Minimalnorm von $\mathfrak{A}$ über $\bar{k}$ setzt die Minimalnorm von $\mathfrak{A}$ über $k$ fort und werde wieder mit $M N$ bezeichnet.

$\mathrm{Zu}$ unserem $x$ gibt es in $\mathfrak{A}$ ein Element $x^{1 / 2}$, dessen Quadrat $x$ ist (s. z.B. [3], [10], [11]). Für $\left(x^{\dagger}\right)^{-1}$ schreiben wir $x^{-1}$. Wir können jetzt umformen:

$$
M N(x+y)=M N\left(P\left(x^{\frac{1}{1}}\right)\left(e+P\left(x^{-\frac{1}{1}}\right) y\right)\right) \quad \text { (s. (1.8))). }
$$

Nach (1.10) folgt daraus:

$$
M N(x+y)=M N(x) M N\left(e+P\left(x^{-1}\right) y\right) .
$$

Wir wollen zeigen: $\left|M N\left(e+P\left(x^{-1 / 2}\right) y\right)\right| \leqq 1$. Dazu sei eine Hilfsbetrachtung eingeschaltet. $P\left(x^{-1 / 2}\right)$ ist bijektive $\bar{k}$-lineare Abbildung von $\mathfrak{A}$ auf sich und bildet daher $\mathfrak{A}$ auf einen $k$-Vektorraum $\mathfrak{B}:=P\left(x^{-1 / 2}\right) \mathfrak{A}$ von gleicher Dimension ab. Wir wollen $\mathfrak{B}$ näher untersuchen.

Zunächst zeigen wir, daß $\mathfrak{B}$ unter der Multiplikation von Jordanalgebra über $k$ ist. Wir brauchen dazu auf Grund der Formel

$$
u v=\frac{1}{2}\left[(u+v)^{2}-u^{2}-v^{2}\right]
$$

nur nachzuweisen:

$$
u \in \mathfrak{B} \Rightarrow u^{2} \in \mathfrak{B} .
$$

Das ergibt sich mit Hilfe der Fundamentalformel (1.4): $\left(P\left(x^{-1 / 2}\right) z\right)^{2}=P\left(P\left(x^{-1 / 2}\right) z\right) e=P\left(x^{-1 / 2}\right) P(z) P\left(x^{-1 / 2}\right) e=P\left(x^{-1 / 2}\right) P(z) x^{-1}$. Liegt $z$ in $\mathfrak{A}$, so liegt auch $P(z) x^{-1}$ in $\mathfrak{A}$.

Diese Jordanalgea $\mathfrak{B}$ enthält das Einselement $e$ :

$$
e=P\left(x^{-1 / 2}\right) x \text {. }
$$

Es ist $\mathfrak{A}=\bar{k} \mathfrak{B}, \operatorname{dim}_{\bar{k}} \mathfrak{A}=\operatorname{dim}_{k} \mathfrak{B}$. Daher ist $\mathfrak{A}$ Grundkörpererweiterung von $\mathfrak{B}$. 
Damit kennen wir die Minimalnorm von $\mathfrak{B}$ über $k$. Sie ist einfach die Restriktion der Minimalnorm $M N$ von $\mathfrak{A}$ über $\bar{k}$.

Mit Hilfe des Kriteriums (1.24) sehen wir jetzt, daß $\mathfrak{B}$ sogar Jordankörper ist: Für $z \neq 0$ aus gilt nach (1.10) und (1.11)

$$
M N\left(P\left(x^{-1 / 2}\right) z\right)=M N(x)^{-1} M N(z) \neq 0 .
$$

Damit sei unsere Hilfsbetrachtung beendet.

Für unser obiges $y$ ist

$$
\left|M N\left(P\left(x^{-1 / 2}\right) y\right)\right|=|M N(x)|^{-1}|M N(y)| \leqq 1,
$$

weil wir $|M N(y)| \leqq|M N(x)|$ voraussetzen konnten. Nach (3.1) ist $w:=P\left(x^{-1 / 2}\right) y$ ganzes Element von $\mathfrak{B}$, also auch $e+w$. (Genügt $w$ einer Gleichung (2.1), so auch $e+w$.) Daher ist $|M N(e+w)| \leqq 1$. Das wollten wir gerade beweisen.

(3.2) liefert nun $|M N(x+y)| \leqq|M N(x)|$.

Wir haben gezeigt:

$$
|M N(x+y)| \leqq \operatorname{Max}(|M N(x)|,|M N(y)|) \quad(x, y \in \mathfrak{A}) .
$$

Aus (3.1) und (3.3) folgt sofort, daß für ganze $x, y$ aus $\mathfrak{A}$ auch $x+y$ ganz ist.

Die Menge der ganzen Elemente von $\mathfrak{A}$ ist ein $A$-Modul, nach Lemma 2.1 also ein Gitter. Es gelten ferner (2.5) und (2.6). q. e.d.

1. Bemerkung. Wir können auf einem lokalen Jordankörper eine Art Bewertung einführen:

Für \| $\|$ gilt

$$
\|x\|:=\sqrt{|M N(x)|}
$$

1. $\|\xi e\|=|\xi|(\xi \in k)$

2. $\|x+y\| \leqq \operatorname{Max}(\|x\|,\|y\|) \quad$ (s. (3.3))

3. $\|P(x) y\|=\|x\|^{2}\|y\| \quad$ (s. (1.10))

4. $\|x y\|=\|x\|\|y\|$,

falls $x, y$ vertauschbar sind oder in einem assoziativen Teilring von $\mathfrak{A}$ liegen.

2. Bemerkung. In assoziativen Divisionsalgebren über diskret und komplett bewertetem $k$ gelten bekanntlich zu Lemma 3.1 und Satz 3.1 gleichlautende Sätze (DruRING [4] S. 100), die sich mit den gleichen Grundgedanken beweisen lassen. Statt (1.10), (3.2) haben wir die einfacher gebauten Formeln:

$M N(x \cdot y)=M N(x) M N(y), \quad M N(x+y)=M N(x) M N\left(e+x^{-1} \cdot y\right)$. 
3. Bemerkung. Die in Satz 3.1 angegebene Maximalordnung genügt im allgemeinen nicht der Bedingung (2.7). Beispiel: $\mathfrak{A}$ sei der Jordankörper $E^{+}$zu der Hamiltonschen Quaternionen-Divisionsalgebra

$$
\rightleftarrows=Q_{2}+Q_{2} i+Q_{2} j+Q_{2} k \quad\left(i^{2}=j^{2}=-1, i j=-j i=k\right)
$$

über dem Körper $Q_{2}$ der 2-adischen Zahlen.

\section{§ 4. Jordanalgebren vom Grad 2}

$\mathfrak{A}$ sei Jordanalgebra vom Grad 2 über dem Quotientenkörper $k$ eines Dedekindringes $A$. (Char. $k \neq 2$ ).

Zunächst einige Bemerkungen zur algebraischen Struktur von $\mathfrak{A}$.

Die Minimalgleichung von $\mathfrak{A}$ lautet:

$$
z^{2}-M S(z) z+M N(z) e=0 .
$$

Es ist $M S(e)=2, M N(e)=1$, denn $e$ hat den zweifachen Eigenwert 1.

$$
\{y, z\}:=M N(y+z)-M N(y)-M N(z)
$$

sei die zu der quadratischen Form $M N(z)$ gehörige Bilinearform. Nach (1.22) ist

$$
M N(z)=1 / 2\left(M S^{2}(z)-M S\left(z^{2}\right)\right)
$$

also

$$
\{y, z\}=M S(y) M S(z)-M S(y z) .
$$

Insbesondere ist

$$
\{y, e\}=2 M S(y)-M S(y)=M S(y) .
$$

Die Multiplikation von $\mathfrak{A}$ läßt sich völlig durch die quadratische Form $M N(z)$ beschreiben.

$$
z^{2}=\{z, e\} z-M N(z) e
$$

liefert nämlich durch Polarisation:

$$
2 y z=\{y, e\} z+\{z, e\} y-\{y, z\} e .
$$

Aus (4.4) liest man sofort ab:

Satz 4.1. Die Isomorphismen einer Jordanalgebra vom Grad 2 sind genau die Isometrien von $\mathfrak{A}$ (bezilitich $M N$ ), die das Einselement festlassen.

Das Bild wird abgerundet durch den folgenden, leicht beweisbaren

Satz 4.2. $\mathfrak{A}$ sei Vektorraum einer Dimension 2 uber $k$. Auf $\mathfrak{A}$ sei eine quadratische Form $F$ und ein Element e mit $F(e)=1$ vorgegeben. 
Dann läßt sich auf $\mathfrak{A}$ in genau einer Weise eine Multiplikation erklären, die $\mathfrak{A}$ zu einer Jordanalgebra vom Grad 2 mit Einselement $e$ und Minimalnorm $F$ macht.

Satz 4.1 und Satz 4.2 zeigen, daß die Theorie der Jordanalgebren vom Grad 2 eine Verfeinerung der Theorie der quadratischen Formen ist, die dadurch bedingt ist, daß ein anisotroper Vektor $e$ ausgezeichnet wird. (Die Bedingung $F(e)=1$ läßt sich immer durch Ubbergang zu einer ähnlichen Form erreichen, ist also nur eine Normierung.)

Ist unser Begriff „Ordnung“" vernünftig gewählt, so müßte er sich in einen brauchbaren Begriff aus der Arithmetik der quadratischen Formen übersetzen lassen. Das wird sich als richtig herausstellen.

Zunächst vermerken wir noch:

Satz 4.3. $\mathfrak{A}$ sei Jordanalgebra vom Grad 2 einer Dimension $\geqq 3$. Dann gilt:

$\mathfrak{A}$ einfach $\Leftrightarrow M N$ nicht ausgeartet (s. z.B. ALBERT [1]).

Wir betrachten ab jetzt nur einfache Algebren vom Grad 2.

Nach Satz 2.1 und (4.2) gilt für ein $z \in \mathfrak{A}$ :

$$
z \operatorname{ganz} \Leftrightarrow\{z, e\} \in A, M N(z) \in A .
$$

Satz 4.4. $\mathfrak{A}$ sei einfach vom Grad 2 uber $k$. $M$ sei Gitter von $\mathfrak{A}$, welches das Einselement enthält. Dann sind folgende Aussagen gleichwertig:

(1) Auf $M$ nimmt $M N$ nur ganze Werte an.

(2) Alle Elemente von $M$ sind ganz.

(3) $x \in M \Rightarrow x^{2} \in M$.

(4) $M$ ist Ordnung.

Beweis.

$(1) \Leftrightarrow(2)$ : Siehe (4.5).

(1) $\Rightarrow(3)$ : Siehe (4.3).

(1) $\Rightarrow(4)$ : Man beachte die Formel

(4.6) $P(x) y=\{x, e\}\{y, e\} x-\{x, y\} x+M N(x) y-M N(x)\{y, e\} e$

die sich aus (4.4) durch direkte Rechnung ergibt.

(4) $\Rightarrow$ (2): Siehe Lemma 2.2.

(3) $\Rightarrow(1)$ : Die Aussagen (1) und (3) genügen dem Lokal-Global-Prinzip. Daher sei $k$ ohne Einschränkung der Allgemeinheit als diskret (und komplett) bewertet vorausgesetzt. Die Funktion $x \rightarrow|M N(x)|$ nimmt auf 128076 Hbg. Math. Abh., Bd. XXVIII 
dem endlich erzdugten $A$-Modul $M$ ihr Maximum an. Wegen $M N\left(x^{2}\right)=$ $M N(x)^{2}$ (s. (1.11)) ist dieses Maximum $\leqq 1$, also $M N(x) \in A$ für alle $x \in M$. q. e. d.

Die maximalen Ordnungen von $\mathfrak{A}$ sind nach Satz 4.4 nichts anderes als die „maximalen Gitter ganzer Norm“ bezüglich $M N$, die das Einselement enthalten.

Abschließend notieren wir, in welches Problem aus der Arithmetik der quadratischen Formen sich die Frage übersetzen läßt, wie viele verschiedene Klassen isomorpher Maximalordnungen eine einfache Jordanalgebra vom Grade 2 besitzt, falls der Grundkörper $k$ diskret und komplett bewertet ist.

Nach EIOHLHR [5], Satz 9.6 sind alle Maximalordnungen von $\mathfrak{A}$ isometrisch. (Die in [5] generell gemachte Voraussetzung, daß der Restklassenkörper von $k$ endlich ist, wird an dieser Stelle nicht gebraucht.) Zwei Maximalordnungen $M_{1}, M_{2}$ von $\mathfrak{A}$ sind nach Satz 4.1 genau dann isomorph, wenn es sogar eine $M_{1}$ in $M_{2}$ überführende Isometrie gibt, die das Einselement $e$ festläßt. Der nächste Satz ist nichts anderes als eine Umformulierung dieses Sachverhaltes.

Zunächst eine

Definition. Zwei Vektoren $x, y$ eines Gitters $M$ eines quadratischen Raumes $\mathfrak{A}$ nennen wir assoziiert, wenn sich eine Isometrie von $\mathfrak{A}$ finden läßt, die $M$ auf $M$ abbildet und $x$ in $y$ überführt.

Assoziierte Vektoren haben gleiche Länge.

Satz 4.5. $\mathfrak{A}$ sei einfache Jordanalgebra vom Grad 2 wber diskret bewertetem kompletten Körper k. M sei ein festgewähltes maximales Gitter ganzer Norm in $\mathfrak{A}$.

Behauptung. Die Klassen isomorpher maximaler Jordanordnungen $\langle B\rangle$ entsprechen eineindeutig den Klassen $\langle z\rangle$ assoziierter Vektoren der Länge 1 von $M$. Eine Zuordnung wird gegeben durch die wohldefinierte Abbildung

$$
\langle B\rangle \rightarrow\langle\varphi(e)\rangle .
$$

Dabei bezeichne $B$ einen beliebig gewählten Vertreter der Klasse isomorpher maximaler Ordnungen, $\varphi$ eine beliebig gewählte Isometrie, die B in $M$ uberfuhrt.

Beweis.

a) Zunächst ist die Abbildung $B \rightarrow\langle\varphi(e)\rangle$ wohldefiniert, weil zwei Isometrien $\varphi_{1}, \varphi_{2}$, die $B$ in $M$ überführen, sich nur um eine Isometrie $\psi$ unterscheiden, die $M$ auf sich abbildet: $\varphi_{2}=\psi \varphi_{1} \cdot \varphi_{2}(e)=\psi\left(\varphi_{1}(e)\right)$ ist zu $\varphi_{1}(e)$ assoziiert. 
Ferner ist für isomorphe maximale Ordnungen $B_{1}, B_{2}$ eine Isometrie $\varphi_{0}$ vorhanden mit $B_{1}=\varphi_{0} B_{2}, \varphi_{0}(e)=e$. Ist $\varphi_{1}$ eine Isometrie, die $B_{1}$ in $M$ überführt, so führt $\varphi_{2}=\varphi_{1} \varphi_{0} B_{2}$ in $M$ über. Bei dieser Wahl ist sogar $\varphi_{2}(e)=\varphi_{1}(e)$, also sicher $\left\langle\varphi_{2}(e)\right\rangle=\left\langle\varphi_{1}(e)\right\rangle$.

b) Die Abbildung (4.7) ist injektiv:

Seien $B_{1}, B_{2}$ maximale Ordnungen, $\varphi_{1}, \varphi_{2}$ Isometrien, die $B_{1}$ bzw. $B_{2}$ in $M$ überführen. Es sei $\varphi_{1}(e)$ zu $\varphi_{2}(e)$ assoziiert. Wir können dann $\varphi_{8}$ so abändern, $\operatorname{da} \beta \varphi_{2}(e)=\varphi_{1}(e)$ wird. $\varphi_{\mathbf{2}}^{-1} \varphi_{1}$ ist ein Automorphismus von $\mathfrak{A}$, der $B_{1}$ in $B_{\mathbf{2}}$ überführt.

c) Die Abbildung (4.7) ist surjektiv:

Sei $g \in M$ ein Vektor der Länge 1. Nach dem Satz von Witt existiert eine Isometrie $\varphi$ mit $\varphi(e)=g$.

$B:=\varphi^{-1}(M)$ ist eine maximale Ordnung.

$\langle B\rangle$ wird vermöge (4.7) auf $\langle g\rangle$ abgebildet. q. e. d.

Nach Eichlare [5] Satz 10.4 ist - zumindest, falls der Restklassenkörper von $k$ endlich ist - die Anzahl der Klassen isomorpher maximaler Ordnungen von $\mathfrak{A}$ endlich. Wir wollen an dieser Stelle auf das Problem der Bestimmung dieser Anzahl nicht näher eingehen (s. [14]).

\section{Literatur}

[1] A. A. Albert, A structure theory for Jordan algebras, Ann. of Math. 48, 446- 467 (1948).

[2] A. A. Albert, A theory of power associative commutative algebras, Trans. Amer. Math. Soc. 69. 503-527 (1950).

[3] H. BraUn und M. KorCHzR, Noch nicht veröffentlichtes Manuskript über Jordanalgebren und Positivitätsbereiche.

[4] M. Drunna, Algebren, Springer 1935, Chelsea 1948.

[5] M. EICHurer, Quadratische Formen und orthogonale Gruppen, Springer 1952.

[6] N. Jacobson, A theorem on the structure of Jordanalgebras, Proc. Nat. Acad. Sci. US. 42, 140-147 (1956).

[7] N. JACOBson, Some groups of transformations defined by Jordan algebras I, J. reine u. angew. Math. 201, (1959) 178-195.

[8] N. JАCOBsoN, Generic norm of an algebra, Osaka Math. J. 15 (1963) 25-50.

[9] M. KNrebsoH, Eine Klasse von Ordnungen in Jordanalgebren vom Grade 3, diese Zeitschrift.

[10] M. KовСнसR, Eine Charakterisierung der Jordanalgebren, Math. Annalen 148, (1962) 244-256.

[11] M. KOECHER, Jordanalgebras and their applications, Lecture notes Univ. Minnesote, Minneapolis 1962. 
184 Manfred Knebusch, Der Begriff der Ordnung einer Jordanalgebra

[12] M. Kokcher, Analysis in reellen Jordanalgebren, Nachr. Akad. Wiss. Göttingen, Math. Phys. Kl. 1958, 67-74.

[13] F. VAN DER BLIJ and T. A. Springer, The arithmetic of octaves and of the group $G_{2}$, Proc. Kon. Ned. Akad. Wet. A, 62 (=Indag. Math., 21) (1959) $406-418$.

[14] M. KNEBUSCH, Assoziierte Vektoren in maximalen Gittern lokaler quadratischer Räume. Erscheint demnächst.

Eingegangen am 13. 11. 1964 